\title{
COMMON FIXED POINT THEOREMS FOR ALMOST WEAKLY PERIODIC NONEXPANSIVE MAPPINGS
}

\author{
KOK-KEONG TAN ${ }^{1}$
}

\begin{abstract}
The notions of normal structure, (convex) diminishing orbital diameters, regular orbital diameters (r.o.d.) have been generalized into a Hausdorff locally convex space $(X, \tau)$ whose topology $\tau$ is generated by a family $\mathscr{P}$ of seminorms. THForem 1. Let $K \subseteq X$ be nonempty weakly compact convex with normal structure w.r.t. $\mathscr{P}$ and $\mathscr{F}$ be a (not necessarily finite nor commuting) family of almost weakly periodic nonexpansive mappings w.r.t. $\mathscr{P}$ on $K$. Then $\mathscr{F}$ has a common fixed point. THEOREM 2. Let $K \subseteq X$ be nonempty weakly compact convex and $\mathscr{F}$ be a semigroup with identity of almost weakly periodic nonexpansive mappings w.r.t. $\mathscr{P}$ on $K$. If $\mathscr{F}$ has r.o.d. w.r.t. $\mathscr{P}$, then $\mathscr{F}$ has a common fixed point. COROLLARY. If $K \subseteq X$ is nonempty weakly compact convex and $\mathscr{F}=\left\{f_{1}, \cdots, f_{n}\right\}$ is a finite commuting family of pointwise periodic nonexpansive mappings w.r.t. $\mathscr{P}$ on $K$, then $F$ has a common fixed point.
\end{abstract}

1. Preliminaries and notations. We shall denote by $\mathscr{Z}^{+}$the set of all nonnegative integers, $\mathscr{N}$ the set of all natural numbers. If $X$ is a nonempty set and $f, g: X \rightarrow X$, we denote $f^{0}=I$, the identity mapping on $X, f g=f \circ g$, the composition of $f$ and $g$, and $f^{n+1}=f\left(f^{n}\right)$, for each $n \in \mathscr{Z}+$. If $x \in X$ and $n \in \mathscr{Z}^{+}$, we denote $O(f, n, x)=\left\{f^{n+\dot{k}}(x): k \in \mathscr{Z}^{+}\right\}$. If $\mathscr{F}$ is a family of mappings on $X$, for each $x \in X$ and each $f \in \mathscr{F}$, we denote $\mathscr{F}(x)=$ $\{g(x): g \in \mathscr{F}\}$ and $\mathscr{F} f(x)=\{g f(x): g \in \mathscr{F}\}$. If $d$ is a pseudometric or a seminorm on $X$, and $A \subseteq X$ is nonempty, $d(A)$ denotes the diameter of $A$ w.r.t. $d$. If $\left({ }^{*}\right)$ is a property, then a mapping $f$ or a family $\mathscr{F}$ of mappings on $X$ is said to have $\left({ }^{*}\right)$ iff $f$ or $\mathscr{F}$ has $\left(^{*}\right)$ at $x$ for each $x \in X$. If $(X, \tau)$ is a topological vector space and $A \subseteq X$, we denote by $\operatorname{Co}(A)$ the convex hull of $A$ and $\bar{C}$ o $(A)$ the closed convex hull of $A$.

Received by the editors July 19, 1971.

AMS 1970 subject classifications. Primary 46.405, 47H10; Secondary 54H25.

Key words and phrases. Almost weakly periodic, weakly periodic, pointwise periodic, periodic, nonexpansive mappings, diminishing orbital diameters, convex diminishing orbital diameters, regular orbital diameters, normal structure.

${ }^{1}$ Part of this work was done while the author was a fellow at the 1971 Summer Research Institute of the Canadian Mathematical Congress, University of Alberta, Edmonton, Alberta. 
Definition 1.1. Let $(X, d)$ be a metric space and $f: X \rightarrow X$. Then $f$ is said to have diminishing orbital diameters at $x \in X$ iff either $f(x)=x$ or there exists an $n \in \mathscr{V}$ such that $d(O(f, n, x))<d(O(f, 0, x))$.

The above notion was first introduced by L. P. Belluce and W. A. Kirk in [1]. The following are some of its generalizations.

Definition 1.2. Let $(X, \tau)$ be a Hausdorff (completely regular) space whose topology $\tau$ is generated by a family $\left\{d_{\lambda}\right\}_{i \in \Lambda}$ of pseudometrics on $X$. (i) If $f: X \rightarrow X$, then $f$ is said to have diminishing orbital diameters (d.o.d.) w.r.t. $\left\{d_{; j i \in \Lambda}\right.$ at $x \in X$ iff either $f(x)=x$ or there exist a $\lambda \in \Lambda$ and an $n \in V^{\prime}$ such that $d_{j}(O(f, n, x))<d_{j}(O(f, 0, x))$. (ii) If $\mathscr{F}$ is a semigroup (under composition) with identity of mappings on $X$, then $\mathscr{F}$ is said to have d.o.d. w.r.t. $\left\{d_{\lambda}\right\}_{\lambda \in \Lambda}$ at $x \in X$ iff either $\mathscr{F}(x)=\{x\}$ or there exist a $\lambda \in \Lambda$ and an $f \in \mathscr{F}$ such that $d_{\lambda}(\mathscr{F} f(x))<\dot{d}_{\lambda}(\mathscr{F}(x))<\infty$.

Definition 1.2(i) is introduced by the author in [5]. In case $\left\{d_{j}\right\}_{i \in \Lambda}$ contains only a single metric, Definition 1.2 (ii) is introduced by M. T. Kiang in [4]. If the domain under consideration is convex, we have the following:

DefINITION 1.3. Let $(X, \tau)$ be a Hausdorff locally convex space whose topology $\tau$ is generated by a family of seminorms on $X, K \subseteq X$ be nonempty closed convex and $\mathscr{F}$ be a semigroup with identity of mappings on $K$. For each $x \in K$, let $C(\mathscr{F}, x)$ be the smallest closed convex subset of $K$ containing $x$ which is $\mathscr{F}$-invariant (i.e. invariant under each $f \in \mathscr{F}$ ). Then (i) $\mathscr{F}$ is said to have convex diminishing orbital diameters (c.d.o.d.) w.r.t. $\mathscr{P}$ at $x \in K$ iff either $\mathscr{P}(x)=\{x\}$ or there exist a $p \in \mathscr{P}$ and an $x_{0} \in \overline{\mathrm{C}} \mathrm{O}(\mathscr{F}(x))$ such that $p\left(\mathscr{F}\left(x_{0}\right)\right)<p(\mathscr{F}(x))<\infty$; (ii) $\mathscr{F}$ is said to have regular orbital diameters (r.o.d.) w.r.t. $\mathscr{P}$ at $x \in K$ iff either $\mathscr{F}(x)=\{x\}$ or there exist a $p \leq \mathscr{P}$ and $y, z \in C(\mathscr{F}, x)$ such that $\sup \{p(y-f(z)): f \in \mathscr{F}\}<$ $p(C(\bar{F}, x))$.

It is clear that $\mathscr{F}$ has d.o.d. w.r.t. $\mathscr{P}$ at $x$ implies $\mathscr{F}$ has c.d.o.d. w.r.t. $\mathscr{P}$ at $x$ which in turn implies $\mathscr{F}$ has r.o.d. w.r.t. $\not \supset$ at $x$. In case only a single norm, Definition 1.3(i) reduces to the notion of c.d.o.d. defined by M. T. Kiang in [4]. Also if $\mathscr{F}=\left\{f^{n}: n \in \mathscr{Z}^{+}\right\}$is the semigroup with identity generated by a single mapping $f$ on $K$, Definition 1.3(ii) reduces to the notion of r.o.d. for a single mapping defined by C. S. Wong in [7].

From now on $(X, \tau)$ denotes a Hausdorff locally convex space whose topology $\tau$ is generated by a family $\mathscr{P}$ of seminorms on $X$.

Definition 1.4. $K \subseteq X$ is said to have normal structure w.r.t. $\mathscr{P}$ iff for any bounded convex subset $H$ of $K$, if $H$ contains more than one point, then there exist a $p \in \mathcal{P}$ and an $x_{0} \in H$ such that $\sup \left\{p\left(x_{0}-x\right): x \in H\right\}<$ $p(H)$.

The notion of normal structure was first introduced by M. S. Brodskii 
and D. P. Milman in [2]. The above generalization was obtained independently by R. D. Holmes and A. Lau in [3] and by the author in [6]. It is known that:

LEMMA 1.5. If $K \subseteq X$ is compact convex, then $K$ has normal structure w.r.t. $\mathscr{P}$.

If $K \subseteq X$ is nonempty closed convex and $\mathscr{F}$ is a family of mappings on $K$, it is easy to see that $\operatorname{Co}(\mathscr{F}(x))$ has normal structure w.r.t. $\mathscr{P}$ implies $\mathscr{F}$ has r.o.d. at $x$, for each $x \in K$.

\section{Common fixed point theorems.}

Definition 2.1. Let $K \subseteq X$ be nonempty and $f: K \rightarrow K$. Then (i) $f$ is almost weakly periodic iff $x \in \overline{\mathrm{C}} \mathrm{o}(O(f, 1, x))$, for each $x \in K$; (ii) $f$ is weakly periodic iff for each $x \in K$, there is a subsequence $\left\{f^{n_{i}}(x)\right\}_{i=1}^{\infty}$ of $\left\{f^{n}(x)\right\}_{n=1}^{\infty}$ such that $f^{n_{i}}(x) \rightarrow x$ weakly; (iii) $f$ is almost pointwise periodic iff for each $x \in K, x \in \bigcap_{n=1}^{\infty} \overline{\mathrm{C}} \mathrm{o}\left(O\left(f^{n}, 1, x\right)\right)$; (iv) $f$ is pointwise periodic iff for each $x \in K$, there is an $N(x) \in \mathscr{N}^{N}$ such that $f^{N}(x)=x ;$ (v) $f$ is periodic iff there is an $N \in \mathscr{N}$ such that $f^{N}=I$, the identity mapping on $K$. (vi) $f$ is nonexpansive w.r.t. $\mathscr{P}$ iff for each $p \in \mathscr{P}, p(f(x)-f(y)) \leqq p(x-y)$, for all $x, y \in K$.

Definition 2.1 (vi) was introduced independently by R. D. Holmes and A. Lau in [3] and by the author in [6].

It is clear that $f$ is periodic implies $f$ is pointwise periodic, $f$ is pointwise periodic implies $f$ is almost pointwise periodic and weakly periodic, and $f$ is weakly periodic (respectively almost pointwise periodic) implies $f$ is almost weakly periodic. Also $f$ is nonexpansive w.r.t. $\mathscr{P}$ implies $f$ is continuous.

THEOREM 2.2. Let $K \subseteq X$ be nonempty weakly compact convex, $\mathscr{F}$ be a (not necessarily finite nor commuting) family of almost weakly periodic nonexpansive mappings w.r.t. $\mathscr{P}$ on $K$ and $\mathscr{S}$ be the semigroup with identity generated by $\mathscr{F}$. Suppose for each $x \in K, \overline{\operatorname{Co}}(\mathscr{S}(x))$ has normal structure w.r.t. $\mathscr{P}$. Then $\mathscr{F}$ has a common fixed point.

Proof. By weak compactness of $K$ and by Zorn's lemma, let $K_{1}$ be minimal w.r.t. being a nonempty closed convex subset of $K$ which is $\mathscr{F}$. invariant. Suppose there exist an $r \in K_{1}$ and an $f_{0} \in \mathscr{F}$ such that $f_{0}(x) \neq x$. Then $\mathscr{S}(x)$ and hence $\overline{\mathrm{C}} \mathrm{o}(\mathscr{S}(x))$ contains more than one point. Thus there exist a $p \in \mathscr{P}$ and an $x_{0} \in \overline{\mathrm{C}} \mathrm{o}(\mathscr{S}(x))$ such that

Define

$$
r_{0}=\sup \left\{p\left(x_{0}-z\right): z \in \overline{\mathrm{C}} \mathrm{o}(\mathscr{S}(x))\right\}<p(\overline{\mathrm{C}} \mathrm{o}(\mathscr{S}(x))) .
$$

$$
M=\left\{y \in K_{1}: \sup \{p(y-z): z \in \mathscr{S}(x)\} \leqq r_{0}\right\} .
$$


Then $M \neq \varnothing$ since $x_{0} \in M$. It is also clear that $M$ is closed and convex. We shall show that $M$ is $\mathscr{F}$-invariant. Indeed let $f \in \mathscr{F}$ and $y \in M$. If $z \in \mathscr{S}(x)$, then $z \in \overline{\mathrm{C}} \mathrm{o}(O(f, 1, z))$ and so for any $\varepsilon>0$, there is a $z_{1} \in$ $\operatorname{Co}(O(f, 1, z))$ with $p\left(z-z_{1}\right)<\varepsilon$. But then $z_{1}=\sum_{i=1}^{m} \lambda_{i} f^{n_{i}}(z)$ for some $n_{i} \geqq 1$ and $0 \leqq \lambda_{i} \leqq 1$ for each $i=1, \cdots, m$ with $\sum_{i=1}^{m} \lambda_{i}=1$. Since $f^{n_{i}-1}(z) \in \mathscr{S}(x)$ for each $i=1, \cdots, m$, we have $p\left(y-f^{n_{i}-1}(z)\right) \leqq r_{0}$, for each $i=1, \cdots, m$. Thus for each $\varepsilon>0$,

$$
\begin{aligned}
p(f(y)-z) & \leqq p\left(f(y)-z_{1}\right)+p\left(z_{1}-z\right)<p\left(f(y)-\sum_{i=1}^{m} \lambda_{i} f^{n_{i}}(z)\right)+\varepsilon \\
& \leqq \sum_{i=1}^{m} \lambda_{i} p\left(f(y)-f^{n_{i}}(z)\right)+\varepsilon \leqq \sum_{i=1}^{m} \lambda_{i} p\left(y-f^{n_{i}-1}(z)\right)+\varepsilon \\
& \leqq \sum_{i=1}^{m} \lambda_{i} r_{0}+\varepsilon=r_{0}+\varepsilon
\end{aligned}
$$

so that $p(f(y)-z) \leqq r_{0}$. Hence $f(y) \in M$, for each $f \in \mathscr{F}$ and each $y \in M$; i.e. $M$ is $\mathscr{F}$-invariant. By minimality of $K_{1}$, we must have $M=K_{1}$. Since $r_{0}<p\left(\overline{\mathrm{C}} \mathrm{o}(\mathscr{S}(x))\right.$ there exist $a, b \in \overline{\mathrm{C}} \mathrm{o}(\mathscr{S}(x))$ such that $p(a-b)>r_{0}$. But then either $a \notin M$ or $b \notin M$, which is a contradiction. Therefore we must have $f(x)=x$ for each $f \in \mathscr{F}$ and each $x \in K_{1}$. Since $K_{1} \neq \varnothing, \mathscr{F}$ has a common fixed point.

In case $\mathscr{F}$ is a semigroup with identity, we have the following slight generalization of the above theorem:

THEOREM 2.3. Let $K \subseteq X$ be nonempty weakly compact convex and $\mathscr{F}$ be a semigroup with identity of almost weakly periodic nonexpansive mappings w.r.t. $\mathscr{P}$ on $K$. If $\mathscr{F}$ has r.o.d. w.r.t. $\mathscr{P}$, then $\mathscr{F}$ has a common fixed point.

Proof. By weak compactness of $K$ and by Zorn's lemma, let $K_{1}$ be minimal w.r.t. being a nonempty closed convex subset of $K$ which is $\mathscr{F}$ invariant. Suppose there exist an $x_{0} \in K_{1}$ and an $f_{0} \in \mathscr{F}$ such that $f_{0}\left(x_{0}\right) \neq$ $x_{0}$. By minimality of $K_{1}$, we must have $C\left(\mathscr{F}, x_{0}\right)=K_{1}$. Since $\mathscr{F}$ has r.o.d. w.r.t. $\mathscr{P}$ and $\mathscr{F}\left(x_{0}\right) \neq\left\{x_{0}\right\}$, there exist $y_{0}, z_{0} \in C\left(\mathscr{F}, x_{0}\right)$ and a $p \in \mathscr{P}$ with $r_{0}=\sup \left\{p\left(y_{0}-f\left(z_{0}\right)\right): f \in \mathscr{F}\right\}<p\left(C\left(\mathscr{F}, x_{0}\right)\right)$. Define

$$
M=\left\{y \in K_{1}: \sup \left\{p\left(y-f\left(z_{0}\right)\right): f \in \mathscr{F}\right\} \leqq r_{0}\right\} .
$$

Then $M$ is nonempty as $y_{0} \in M$. It is clear that $M$ is also closed and convex. Also $M$ can be shown to be $\mathscr{F}$-invariant by the same argument as in the proof of Theorem 2.2. Hence $M=K_{1}$, by the minimality of $K_{1}$. Next we define $N=\left\{z \in K_{1}: p(y-f(z)) \leqq r_{0}\right.$, for all $y \in K_{1}$ and all $\left.f \in \mathscr{F}\right\}$. Then $N$ is nonempty as $z_{0} \in N$. Since each $f \in \mathscr{F}$ is continuous, $N$ is closed. Since $\mathscr{F}$ is a semigroup, $N$ is $\mathscr{F}$-invariant. We shall now show that $N$ is also convex. 
Indeed, let $z_{1}, z_{2} \in N, 0<\lambda<1$, and $z=\lambda z_{1}+(1-\lambda) z_{2}$. Suppose $y \in K_{1}$ and $f \in \mathscr{F}$. Since $y \in \overline{\operatorname{Co}}(O(f, 1, y))$, for each $\varepsilon>0$, there is a $y_{1} \in \operatorname{Co}(O(f, 1, y))$ with $p\left(y-y_{j}\right)<\varepsilon$. But then $y_{1}=\sum_{i=1}^{m} \lambda_{i} f^{n_{i}}(y)$, for some $n_{i} \geqq 1,0 \leqq \lambda_{i} \leqq 1$, for each $i=1, \cdots, m$ with $\sum_{i=1}^{m} \lambda_{i}=1$. Thus for each $\varepsilon>0$,

$$
\begin{aligned}
p(y-f(z)) & \leqq p\left(y-y_{1}\right)+p\left(y_{1}-f(z)\right)<\varepsilon+p\left(\sum_{i=1}^{m} \lambda_{i} f^{n_{i}}(y)-f(z)\right) \\
& \leqq \varepsilon+\sum_{i=1}^{m} \lambda_{i} p\left(f^{n_{i}}(y)-f(z)\right) \\
& \leqq \varepsilon+\sum_{i=1}^{m} \lambda_{i} p\left(f^{n_{i}-1}(y)-\left(\lambda z_{1}+(1-\lambda) z_{2}\right)\right) \\
& \leqq \varepsilon+\sum_{i=1}^{m} \lambda_{i}\left[\lambda p\left(f^{n_{i}-1}(y)-z_{1}\right)+(1-\lambda) p\left(f^{n_{i}-1}(y)-z_{2}\right)\right] \\
& \leqq \varepsilon+\sum_{i=1}^{m} \lambda_{i}\left[\lambda r_{0}+(1-\lambda) r_{0}\right]=\varepsilon+\sum_{i=1}^{m} \lambda_{i} r_{0}=\varepsilon+r_{0},
\end{aligned}
$$

so that $p(y-f(z)) \leqq r_{0}$, for all $y \in K_{1}$ and all $f \in \mathscr{F}$. Hence $z=\lambda z_{1}+$ $(1-\lambda) z_{2}$ is in $N$ and therefore $N$ is convex. By minimality of $K_{1}$ again, $K_{1}=N$. Since $r_{0}<p\left(C\left(\mathscr{F}, x_{0}\right)\right)=p\left(K_{1}\right)$, there are $a, b \in K_{1}$ with $p(a-b)>r_{0}$. Since $I \in \mathscr{F}$, it follows that neither $a$ nor $b$ is in $N$, which is a contradiction. Therefore $f(x)=x$ for each $x \in K_{1}$ and each $f \in \mathscr{F}$. Since $K_{1}$ is nonempty, $\mathscr{F}$ has a common fixed point.

COROLlARY 2.4. Let $K \subseteq X$ be nonempty weakly compact convex with normal structure w.r.t. $\mathscr{P}$ and $\mathscr{F}$ be any (not necessarily finite nor commuting) family of almost weakly periodic nonexpansive mappings w.r.t. $\mathscr{P}$ on $K$. Then $\mathscr{F}$ has a common fixed point.

COROLLARY 2.5. Let $K \subseteq X$ be nonempty weakly compact convex and $\mathscr{F}$ be a semigroup with identity of almost weakly periodic nonexpansive mappings w.r.t. $\mathscr{P}$ on K. If $\mathscr{F}$ has c.d.o.d. w.r.t. $\mathscr{P}$ then $\mathscr{F}$ has a common fixed point.

COROLlARY 2.6. Let $K \subseteq X$ be nonempty weakly compact convex and $\mathscr{F}=\left\{f_{1}, \cdots, f_{n}\right\}$ be a finite commuting family of pointwise periodic nonexpansive mappings w.r.t. $\mathscr{P}$ on $K$. Then $\mathscr{F}$ has a common fixed point.

Proof. Let $\mathscr{S}$ be the semigroup with identity generated by $\mathscr{F}$. Then for each $x \in K, \mathscr{S}(x)$ is a finite subset of $K$ and so $\mathrm{Co}(\mathscr{S}(x))$ is compact convex. By Lemma 1.5, $\overline{\mathrm{C}}(\mathscr{S}(x))$ has normal structure w.r.t. $\mathscr{P}$ (and hence r.o.d. w.r.t. $\mathscr{P}$ ) so that by Theorem 2.2 (or by Theorem 2.3), $\mathscr{F}$ has a common fixed point. 
COROLlaRY 2.7. Let $K \subseteq X$ be nonempty compact convex and $\mathscr{F}$ be any (not necessarily finite nor commuting) family of almost weakly periodic nonexpansive mappings w.r.t. $\mathscr{P}$ on $K$. Then $\mathscr{F}$ has a common fixed point.

Since every uniformly convex Banach space (in particular, every Hilbert space) has normal structure, we have the following observation: if $K$ is any nonempty weakly compact (or equivalently bounded closed) convex subset of a uniformly convex Banach space $X$, and if there is an almost weakly periodic nonexpansive mapping on $K$ which has a unique fixed point $x_{0} \in K$, then $x_{0}$ is fixed under any other almost weakly periodic (respectively weakly periodic, almost pointwise periodic, pointwise periodic or periodic) nonexpansive mapping on $K$. In particular, if $K$ is the closed unit ball $\{x \leqq X:\|x\| \leqq 1\}$, then $f(0)=0$, for any almost weakly periodic nonexpansive mapping $f$ on $K$. Indeed, define $f_{0}(x)=-x$, for each $x \in K$, then $f_{0}$ is periodic and isometric (and hence nonexpansive), and 0 is the unique fixed point of $f_{0}$.

REMARK 2.8. Even when $K$ is a nonempty weakly compact convex subset of a Banach space $X$, it is not known whether a countably infinite commuting family $\mathscr{F}$ of periodic nonexpansive mappings on $K$ will have a common fixed point.

\section{REFERENCES}

1. L. P. Belluce and W. A. Kirk, Fixed-point theorems for certain classes of nonexpansive mappings, Proc. Amer. Math. Soc. 20 (1969), 141-146. MR 38 \#1663.

2. M. S. Brodskii and D. P. Mil' man, On the center of a convex set, Dokl. Akad. Nauk SSSR 59 (1948), 837-840. (Russian) MR 9, 448.

3. R. D. Holmes and A. Lau, Nonexpansive actions of topological semigroups and fixed points, J. London Math. Soc. (to appear).

4. M. T. Kiang, Remetrizations and common fixed point theorems for semigroup of mappings, Ph.D. Thesis, Dalhousie University, 1971.

5. K. K. Tan, Fixed point theorems for nonexpansive mappings, Seminar on topology and functional analysis, Dalhousie University, 1970. (Part of the results is to appear in Pacific J. Math. under the same title while the rest is still in preparation for publication.) 6. - Some fixed point theorems for nonexpansive mappings in Hausdorfflocally convex spaces, Ph.D. Thesis, University of British Columbia, 1970.

7. C. S. Wong, Fixed point theorems (to appear).

Department of Mathematics, Dalhousie University, Halifax, Nova Scotia, CANADA 